W. Grudziński, B. Koszela, T. T. Świạtkowski, Institute of Mathematics, Technical University of Łódź, 90-924 Łódź, Poland.

W. Wilczyński, Institute of Mathematics, University of Łódź, 90-238 Łódź,

Poland, email:wwil@@krysia.uni.lodz.pl

\title{
CLASSES OF CONTINUOUS REAL FUNCTIONS (II)
}

This survey will consist of four parts. The topological terminology used in this paper is consistent with that found in Engelking ([1]).

Studies comparing the classes of the continuous functions by different topologies were run in Łódź since the early seventies. The subject matter of these studies and the results achieved between $1973-1978$ have been discussed in paper [8]. The present paper presents the results achieved in the years $1978-1995$.

\section{Topological Extensions Not Changing the Class of Con- tinuous Functions}

Most of the results of this section can be found in [2]. Let $T_{o}$ and $T$ be topologies on a set $X$. Let $C\left(X, T_{o}\right)$ and $C(X, T)$ denote the classes of the $T_{o}$ and $T$-continuous functions, respectively, defined on the set $X$, whose values belong to some arbitrarily fixed metric space $(Y, d)$.

In theorems in which the classes of the continuous functions in a given topology are mentioned, we will most often not mention their set of values, having in mind then some fixed metric space $(Y, d)$ consisting of more than one point.

Definition 1 A topology $T$ will be called an extension of topology $T_{o}$ in a set $X$, if $T_{o} \subset T$.

\footnotetext{
Key Words: generalized continuity, countably compact, pseudocompact, $\star$-compact

Mathematical Reviews subject classification: Primary: 54C05 Secondary:26A21

Received by the editors March 19, 1996

*Professor Swiątkowski passed away on October 30, 1994 while in the process of com-
} pleting the work for this article 
The interiors and the closures of a set $A \subset X$ in a topology $T$ will be denoted by $\operatorname{Int}_{T} A$ and $\mathrm{Cl}_{T} A$ respectively. If $T$ is an extension of a topology $T_{o}$ in a set $X$, then naturally for any $A \subset X$ we have that $\operatorname{Int}_{T_{o}} A \subset \operatorname{Int}_{T} A$ and $\mathrm{Cl}_{T} A \subset \mathrm{Cl}_{T_{o}} A$.

A neighborhood of a point $p \in X$ will be denoted by $A_{p}, B_{p}, V_{p}$, etc. Writing $A_{p}\left(A_{p} \in T\right)$ we signal that $A_{p}$ is a $T$-neighborhood of $p$. The following theorem was proved in [3].

Theorem 1 Let $T$ be an extension of the natural topology $T_{o}$ on an interval $[a, b] \subset \mathbb{R}$. If

(C) $C\left([a, b], T_{o}\right)=C([a, b], T)$,

then

(G) if $x \in[a, b]$ and $A_{x}$ is a T-neighborhood of $x$, then $A_{x}$ is $T_{o}$-dense in some $T_{o}$-neighborhood of $x$, and

$\left(S_{1}\right)$ every interval $[c, d] \subset[a, b]$ is T-connected.

Later we find conditions sufficient for $(C)$ in a general setting. Among others, we use conditions similar to conditions $(G)$ and $\left(S_{1}\right)$ from Theorem 1. The following lemma will make it possible to replace condition $(G)$ with a condition which is easier to check.

Lemma 1 Let $T$ be an extension of a topology $T_{o}$ in a set $X$. The following conditions are equivalent:

(G) $\forall_{x \in X} \forall A_{x} \in T \quad\left[A_{x}\right.$ is a $T_{o}$-dense set in some $T_{o}$-neighborhood of a point $x]$

(H) $\forall$ A $\in$ T $A \subset \operatorname{Int}_{T_{o}} \mathrm{Cl}_{T_{o}} A$.

$\operatorname{ProOF}$ OF $(G) \Rightarrow(H)$.

Let $A \in T$. If $A=\emptyset$, then naturally $A \subset \operatorname{Int}_{T_{o}} \mathrm{Cl}_{T_{o}} A$. However if $A \neq \emptyset$, then $A$ is a $T$ - neighborhood of each of its points. Thus, by $(G)$, for every $x \in A$ there exists a $T_{o}$-neighborhood $G_{x}$ such that $G_{x} \subset \mathrm{Cl}_{T_{o}} A$. Hence we have $A \subset \bigcup_{x \in A} G_{x} \subset \mathrm{Cl}_{T_{o}} A$ and so

$$
A \subset \bigcup_{x \in A} G_{x} \subset \operatorname{Int}_{T_{o}} \mathrm{Cl}_{T_{o}} A .
$$

Proof of $(H) \Rightarrow(G)$. Let $A \in T$. Because of $(H)$ we have $A \subset \operatorname{Int}_{T_{o}} \mathrm{Cl}_{T_{o}} A \subset$ $\mathrm{Cl}_{T_{o}} A$. Thus the set $A$ is $T_{o}$-dense in a $T_{o}$-open set $\operatorname{Int}_{T_{o}} \mathrm{Cl}_{T_{o}} A$. 
Although Theorem 1 is formulated for closed intervals in $\mathbb{R}$, its proof uses only the connectedness of intervals and not their compactness. Hence, from Lemma 1 we have the following assertion.

Theorem 2 Let $T$ be an extension of a topology $T_{o}$ in a $T_{o}$-connected subset $X \subset \mathbb{R}$. If

(C) $C\left(X, T_{o}\right)=C(X, T)$,

then

(H) $\forall_{A \in T} \quad A \subset \operatorname{Int}_{T_{o}} \mathrm{Cl}_{T_{o}} A$, and

(S) $T$ does not decrease the class of $T_{o}$-connected sets.

Definition 2 Let $(X, T)$ be a topological space and let $W \subset X$. The smallest topology containing both $T$ and $W$ will be called the simple extension of $T$ generated by the set $W$ and will be denoted by $T(W)$.

Obviously, $T=T(W)$ if and only if $W \in T$. It is easy to observe that

$$
T(W)=\left\{A \in 2^{X}: \exists_{U, V \in T} \quad W=(U \cap W) \cup V\right\}
$$

Lemma 2 Let $(X, T)$ be a topological space and $T(W)$ a simple extension of $T$. The following conditions are equivalent.

(H) $\forall_{A \in T(W)} A \subset \operatorname{Int}_{T} \mathrm{Cl}_{T} A$.

(i) $W \subset \operatorname{Int}_{T} \mathrm{Cl}_{T} W$.

Theorem 3 The simple extensions of the topology which satisfy condition $(H)$ do not change the class of the continuous functions in a given topological space.

Proof. Let $(X, T)$ be a topological space and let $W \subset X$. Obviously, $C(X, T) \subset C(X, T(W))$. Assume that the opposite inclusion does not hold. Then there exists a $T(W)$-continuous and $T$-discontinuous function $f: X \rightarrow Y$. Let $p \in X$ be a point of $T$-discontinuity of $f$. Then

$$
\exists_{\varepsilon>0} \forall_{U_{p} \in T} \exists_{x \in U_{p}} d(f(x), f(p)) \geq \varepsilon .
$$

Because of the $T(W)$-continuity of $f$ at $p$, for $\varepsilon / 2$ there exists a $T(W)$ neighborhood, $\left.A_{p}=(U \cap W) \cup V\right)$ of $p$, where $U, V \in T$ and for which

$$
\forall_{x \in A_{p}} d(f(x), f(p))<\varepsilon / 2
$$


Observe that $p \in U \cap W$, otherwise $p \in V \in T$ and (2) contradicts (1). So using condition $(H)$ we have that $p \in U \cap W \subset W \subset \operatorname{Int}_{T} \mathrm{Cl}_{T} \mathrm{~W}$. Using (1) it now follows that $U_{p}=U \cap \operatorname{Int}_{T} \mathrm{Cl}_{T} W \in T$ so that

$$
\exists_{s \in U \cap \operatorname{Int}_{T} \mathrm{Cl}_{T} W} d(f(s), f(p)) \geq \varepsilon .
$$

From this and (2) it follows that $s \notin W$. As $f$ is $T(W)$-continuous at $s$ we have

$$
\exists_{A_{s}=\left(U^{\prime} \cap W\right) \cup V^{\prime} \in T(W)} \forall_{x \in A_{s}} d(f(x), f(s))<\varepsilon / 2,
$$

where $U^{\prime}$ and $V^{\prime}$ are $T$-open. Since $s \notin W$, this implies that $s \in V^{\prime}$. As $U$ is $T$-open, $U \cap \mathrm{Cl}_{T} W \subset \mathrm{Cl}_{T}(U \cap W)$. But $s \in U \cap \operatorname{Int}_{T} \mathrm{Cl}_{T} W \subset \mathrm{Cl}_{T} W$ and $s \in V^{\prime} \in T$. So using the above we have that $(U \cap W) \cap V^{\prime} \neq \emptyset$. Let $k \in(U \cap W) \cap V^{\prime}$. Then from (2), (3) and (4) we conclude that

$$
\varepsilon \leq d(f(s), f(p))+d(f(k), f(p))<\varepsilon,
$$

which is a contradiction. Hence, $C(X, T)=C(X, T(W))$ as required.

Remark 1 By using Theorem 3 we can construct whole sequences of topologies larger than a given topology $T$, in which each successive one is a simple extension of the former one and which does not change the class of the T-continuous functions. However, in order for the subsequent simple extension to be different from the preceding one at a given stage of construction, a nonopen set $W$ which satisfies condition $(H)$ must be available.

Remark 2 If $T$ is a maximal (in the sense of inclusion) topology which includes the topology $T_{o}$ and satisfies the condition $C\left(X, T_{o}\right)=C(X, T)$, then

(M) $A \in T \Leftrightarrow A \subset \operatorname{Int}_{T} \mathrm{Cl}_{T}$.

In particular, condition $(M)$ is satisfied if the topology $T$ cannot be extended without changing the continuous functions.

Proof. As $T$-open sets satisfy condition $(H)$, they do not satisfy condition $(M)$. This means that there exists a set $W \in T_{o}$ such that $W \subset \operatorname{Int}_{T} \mathrm{Cl}_{T} W$ and $W \notin T$. In light of Theorem 3, the topology $T(W)$ preserves the class of $T$-continuous functions and is larger than $T$, whenever $W \in T(W)$.

Remark 3 Theorem 3 cannot generally be reversed; i.e. condition $(H)$ does not follow from the condition $C\left(X, T_{o}\right)=C(X, T)$ even if $T$ is a simple extension of $T_{o}$. 
Example 1 Let $X=[0,1)$. The family $T_{o}=\{[0, a)\}$ is a topology in $X$. Let $T=T_{0}(\{1 / 2\})$. Constant functions are the only $T_{o}$-continuous real functions here, and consequently, $C\left(X, T_{o}\right)=C(X, T)$. It is easy to see that $\mathrm{Cl}_{T_{o}}\{1 / 2\}=[1 / 2,1)$ and $\operatorname{Int}_{T_{o}}([1 / 2,1))=\emptyset$. Thus, the set $\{1 / 2\}$ does not satisfy condition $(H)$.

Remark 4 If $T$ is a simple extension of the natural topology $T_{o}$ on a $T_{o^{-}}$ connected subset $X \subset \mathbb{R}$, then by Theorems 1 and 3 conditions $(H)$ and $(C)$ are equivalent. In particular we get the following.

Theorem 4 Let $T$ be a simple extension of the natural topology $T_{o}$ on the set of real numbers $\mathbb{R}$. Then the following conditions are equivalent.

(H) $\forall_{a \in T} A \subset \operatorname{Int}_{T_{o}} \mathrm{Cl}_{T_{o}} A$.

(C) $C\left(X, T_{o}\right)=C(X, T)$.

Theorem 5 Let $T$ be an arbitrary extension of a topology $T_{o}$ on the set $X$. If

$$
\text { (K) } \forall_{A \in T} A \subset \operatorname{Int}_{T_{o}} \mathrm{Cl}_{T} A \text {, }
$$

then

$$
\text { (C) } C\left(X, T_{o}\right)=C(X, T) \text {. }
$$

Proof. Obviously, $C\left(X, T_{o}\right) \subset C(X, T)$. Let $f \in C(X, T)$ and $p \in X$. We will show that $f$ is $T_{o}$-continuous at $p$. Let $\varepsilon>0$. As $f$ is $T$-continuous at $p$, $\exists_{A_{p} \in T} \forall_{x \in A_{p}} d(f(p), f(x))<\varepsilon / 2$ where $A_{p}$ is a $T$-neighborhood of $p$. From assumption $(K)$ we have $p \in A_{p} \subset \operatorname{Int}_{T_{o}} \mathrm{Cl}_{T} A_{p}$. We complete the proof by showing

$$
\forall x \in \operatorname{Int}_{T_{o}} \mathrm{Cl}_{T} A_{p} d(f(x), f(p))<\varepsilon .
$$

Let $x \in \operatorname{Int}_{T_{o}} \mathrm{Cl}_{T} A_{p}$. As $f$ is $T$-continuous at $x$, there is a $T$-neighborhood $A_{x}$ of $x$ such that $\forall_{y \in A_{x}} d(f(x), f(y))<\varepsilon / 2 \quad \mathrm{As}_{\mathrm{Int}} \mathrm{I}_{o} \mathrm{Cl}_{T} A_{p} \subset \mathrm{Cl}_{T} A_{p}$, $x \in \mathrm{Cl}_{T} A_{p}$ and in particular $A_{p} \cap A_{x} \neq \emptyset$. Let $z \in A_{p} \cap A_{x}$. From the definitions of $A_{p}$ and $A_{x}$ we obtain

$$
d(f(p), f(x)) \leq d(f(p), f(z))+d(f(z), f(p))<\varepsilon,
$$

which completes proof of (5). As $f$ is $T_{o}$-continuous at an arbitrary point $x \in X,(C)$ holds.

Remark 5 In the case, in which $T$ is a simple extension of a topology $T_{o}$, conditions $(C)$ and $(K)$ are equivalent. 
Remark 6 The converse to Theorem 5 is not true. This will be discussed later in this survey.

Definition 3 Let $T$ be an extension of the topology $T_{o}$ on a set $X$. A nonempty set $A \in T$ is called:

- $T$-significant, if $\operatorname{Int}_{T_{o}} \mathrm{Cl}_{T} A=\emptyset$.

- T-strong, if each element of a set $A$ has a T-significant neighborhood.

- T-weak, if it is not T-strong.

- A point $p \in X$ is called T-local, if for every $T$-neighborhood $A_{p}$ of $p$, there exists a set $C$ such that $\emptyset \neq \operatorname{Int}_{T} \mathrm{Cl}_{T} C \subset \mathrm{Cl}_{T} A_{p}$ and $\operatorname{Int}_{T_{o}} \mathrm{Cl}_{T_{o}} C \subset C$

Lemma 3 Let $T$ be an extension of the topology $T_{o}$ on a set $X$ such that

(H) $\forall_{A \in T} A \subset \operatorname{Int}_{T_{o}} \mathrm{Cl}_{T_{o}} A$.

Let $U$ be an arbitrary $T_{o}$-open subset of a set $X$. If

$\left(P_{o}\right)$ in the set $U$ there exists a T-local point,

then

$\left(R_{o}\right)$ the set $U$ is $T$-weak.

If $T_{o}$ is the natural topology in the set of the real numbers, then $\left(R_{o}\right)$ also implies $\left(P_{o}\right)$.

Proof. Let $p$ be a $T$-local point of a set $U$ and $A_{p}$ be a $T$-neighborhood of $p$. Then there exists a set $C$ such that $\emptyset \neq \operatorname{Int}_{T} \mathrm{Cl}_{T} C \subset \mathrm{Cl}_{T} A_{p}$ and $\operatorname{Int}_{T_{o}} \mathrm{Cl}_{T_{o}} C \subset C$.

From this and $(H)$ we obtain:

$$
\begin{gathered}
\emptyset \neq \operatorname{Int}_{T} \mathrm{Cl}_{T} C \subset \operatorname{Int}_{T} \mathrm{Cl}_{T_{o}} C \subset \operatorname{Int}_{T_{o}} \mathrm{Cl}_{T_{o}}\left(\operatorname{Int}_{T} \mathrm{Cl}_{T_{o}} C\right) \subset \\
\subset \operatorname{Int}_{T_{o}} \mathrm{Cl}_{T_{o}}\left(\mathrm{Cl}_{T_{o}} C\right)=\operatorname{Int}_{T_{o}} \mathrm{Cl}_{T_{o}} C \subset C \subset \mathrm{Cl}_{T} C .
\end{gathered}
$$

Hence

$$
\emptyset \neq \operatorname{Int}_{T_{o}} \mathrm{Cl}_{T_{o}} C \subset \mathrm{Cl}_{T} C
$$

and as $T_{o} \subset T$,

$$
\emptyset \neq \operatorname{Int}_{T_{o}} \mathrm{Cl}_{T_{o}} C \subset \operatorname{Int}_{T} \mathrm{Cl}_{T} C .
$$


It now follows from the choice of $C$ that $\emptyset \neq \operatorname{Int}_{T_{o}} \mathrm{Cl}_{T_{o}} C \subset \operatorname{Int}_{T} \mathrm{Cl}_{T} C \subset$ $\mathrm{Cl}_{T} A_{p}$ and hence, that $\operatorname{Int}_{T_{o}} \mathrm{Cl}_{T} A_{p} \neq \emptyset$. This means that no $T$-neighborhood of $p$ is $T$-significant. Hence, $U$ is $T$-weak and the proof of the first part of the lemma is complete.

Now assume that $T_{o}$ is the natural topology on $\mathbb{R}$, and let $U \subset \mathbb{R}$ be a $T$-weak set. Then there exists a point $p \in U$ and a $T$-neighborhood, $A_{p}$, of $p$ such that $\operatorname{Int}_{T_{o}} \mathrm{Cl}_{T} A_{p} \neq \emptyset$. As $\operatorname{Int}_{T_{o}} \mathrm{Cl}_{T} A_{p}$ is open in $\mathbb{R}$, it contains an open interval $B$, (i.e. a $T_{o}$-open set). The set $B$ is $T_{o}$-connected; so $\emptyset \neq \operatorname{Int}_{T_{o}} \mathrm{Cl}_{T} B \subset B$. Because of this and assumption $(H)$ we have

$$
\begin{aligned}
\operatorname{Int}_{T} \mathrm{Cl}_{T} B & \subset \operatorname{Int}_{T} \mathrm{Cl}_{T_{o}} B \subset \operatorname{Int}_{T_{o}} \mathrm{Cl}_{T_{o}} \operatorname{Int}_{T} \mathrm{Cl}_{T_{o}} B \subset \operatorname{Int}_{T_{o}} \mathrm{Cl}_{T_{o}} \mathrm{Cl}_{T_{o}} B \subset \\
& \subset \operatorname{Int}_{T_{o}} \mathrm{Cl}_{T_{o}} B \subset B \subset \mathrm{Cl}_{T} B .
\end{aligned}
$$

As $T_{o} \subset T$,

$$
\operatorname{Int}_{T} \mathrm{Cl}_{T} B \subset \operatorname{Int}_{T_{o}} \mathrm{Cl}_{T_{o}} B \subset \operatorname{Int}_{T} \mathrm{Cl}_{T} B,
$$

and as a result, $\operatorname{Int}_{T} \mathrm{Cl}_{T} B=\operatorname{Int}_{T_{o}} \mathrm{Cl}_{T_{o}} B$. Summarizing we obtain

$$
\emptyset \neq \operatorname{Int}_{T} \mathrm{Cl}_{T} B=\operatorname{Int}_{T_{o}} \mathrm{Cl}_{T_{o}} B \subset B \subset \operatorname{Int}_{T_{o}} \mathrm{Cl}_{T} A_{p} \subset \mathrm{Cl}_{T} A_{p} .
$$

As $A_{p} \in T$ is arbitrary, we find that $p \in U$ is a $T$-local point, and hence, $\mathbb{R}$ with the natural topology is $\left(P_{0}\right)$.

Theorem 6 Let $T$ be an extension of a topology $T_{o}$ on a set $X$. If

(H) $\forall \forall_{A \in T} \quad A \subset \operatorname{Int}_{T_{o}} \mathrm{Cl}_{T_{o}} A$.

$\left(R_{1}\right)$ For an arbitrary $T_{o}$-closed set $F$ the set $\operatorname{Int}_{T_{o}} F$ is $T$-weak,

then

(C) $C\left(X, T_{o}\right)=C(X, T)$.

Proof. Obviously it is enough to show that every $T$-continuous function is $T_{o}$-continuous. Let $f \in C(X, T), p \in X$ and $\varepsilon>0$. From $T$-continuity of $f$ at $p$ we have $\exists_{A_{p} \in T} \forall_{x \in A_{p}} d(f(x), f(p))<\varepsilon / 6$, and due to assumption $(H)$ we have $A_{p} \subset \operatorname{Int}_{T_{o}} \mathrm{Cl}_{T_{o}} A$. Hence, $A_{p}$ is a $T$-neighborhood of $p$. We will show that

$$
\forall x \in \operatorname{Int}_{T_{o}} \mathrm{Cl}_{T_{o}} A_{p} \in T_{o} d(f(x), f(p))<\varepsilon .
$$

which will obviously mean that $f$ is $T_{o}$-continuous at $p$. Let $a \in \operatorname{Int}_{T_{o}} \mathrm{Cl}_{T_{o}} A_{p}$. From $T$-continuity of $f$ at $a$ we have that

$$
\exists_{A_{a} \in T} \forall_{x \in A_{a}} d(f(x), f(a))<\varepsilon / 6 .
$$


The set $\mathrm{Cl}_{T_{o}} A_{p} \cap \mathrm{Cl}_{T_{o}} A_{a}$ is obviously $T_{o}$-closed, while the set $\operatorname{Int}_{T_{o}}\left(\mathrm{Cl}_{T_{o}} A_{p} \cap\right.$ $\mathrm{Cl}_{T_{o}} A_{a}$ ) is nonempty (it contains $a$ ), so by assumption $\left(R_{1}\right)$ there exists a point $y \in \operatorname{Int}_{T_{o}}\left(\mathrm{Cl}_{T_{o}} A_{p} \cap \mathrm{Cl}_{T_{o}} A_{a}\right)$, no $T$-neighborhood of which is $T$ significant. From $T$-continuity of $f$ at $y$ we have $\exists_{A_{y} \in T} \forall_{x \in A_{y}} d(f(x), f(y))<$ $\varepsilon / 6$. Let $B_{y}=A_{y} \cap \operatorname{Int}_{T_{o}}\left(\mathrm{Cl}_{T_{o}} A_{p} \cap \mathrm{Cl}_{T_{o}} A_{a}\right) . B_{y}$ is obviously a $T$-neighborhood of a point $y$; so by $\left(\mathrm{R}_{1}\right)$ it follows that $\operatorname{Int}_{T_{o}} \mathrm{Cl}_{T} B_{y} \neq \emptyset$. Observe that

$$
\begin{gathered}
\operatorname{Int}_{T_{o}} \mathrm{Cl}_{T} B_{y}=\operatorname{Int}_{T_{o}} \mathrm{Cl}_{T}\left[A_{y} \cap \operatorname{Int}_{T_{o}}\left(\mathrm{Cl}_{T_{o}} A_{p} \cap \mathrm{Cl}_{T_{o}} A_{a}\right)\right] \subset \\
\subset \mathrm{Cl}_{T} A_{y} \cap \mathrm{Cl}_{T}\left(\mathrm{Cl}_{T_{o}} A_{p} \cap \mathrm{Cl}_{T_{o}} A_{a}\right) \subset \mathrm{Cl}_{T} A_{y} \cap \mathrm{Cl}_{T_{o}} A_{p} \cap \mathrm{Cl}_{T_{o}} A_{a} .
\end{gathered}
$$

That is,

$$
\emptyset \neq \operatorname{Int}_{T_{o}} \mathrm{Cl}_{T} B_{y} \subset \mathrm{Cl}_{T} A_{y} \cap \mathrm{Cl}_{T_{o}} A_{p} \cap \mathrm{Cl}_{T_{o}} A_{a}
$$

Thus we find that $A_{p} \cap \operatorname{Int}_{T_{o}} \mathrm{Cl}_{T} B_{y} \neq \emptyset$ and $A_{a} \cap \operatorname{Int}_{T_{o}} \mathrm{Cl}_{T} B_{y} \neq \emptyset$. Let $b, c$ be elements of these respective intersections. Using $T$-continuity of $f$ at points $b$ and $c$ we obtain that

$$
\begin{aligned}
& \exists_{A_{b} \in T} \forall_{x \in A_{b}} d(f(x), f(b))<\varepsilon / 6, \\
& \exists_{A_{c} \in T} \forall_{x \in A_{c}} d(f(x), f(c))<\varepsilon / 6 .
\end{aligned}
$$

From (6) it follows that $b \in \mathrm{Cl}_{T} A_{y}$ and $c \in \mathrm{Cl}_{T} A_{y}$. Hence $A_{b} \cap A_{y} \neq \emptyset$ and $A_{c} \cap A_{y} \neq \emptyset$. Let $b^{\prime}$ and $c^{\prime}$ be elements of these respective intersections. It now follows that

$$
\begin{aligned}
d(f(p), f(a)) \leq & d(f(p), f(b))+d\left(f(b), f\left(b^{\prime}\right)\right)+d\left(f\left(b^{\prime}\right), f(y)\right)+ \\
& +d\left(f(y), f\left(c^{\prime}\right)\right)+d\left(f\left(c^{\prime}\right), f(c)\right)+d(f(c), f(a))<\varepsilon .
\end{aligned}
$$

Hence, $f$ is $T_{0}$ continuous at $p$, and as $p$ was arbitrary, the theorem follows.

Remark 7 Suppose the assumptions of Theorem 6 hold and that $a, p, A_{a}$ and $A_{p}$ are as in the proof. If $d(f(x), f(p))<\varepsilon \forall x \in A_{a}$, and $\operatorname{Int}_{T_{o}}\left(\mathrm{Cl}_{T_{o}} A_{p} \cap\right.$ $\left.\mathrm{Cl}_{T_{o}} A_{a}\right)$ is T-weak, then $d(f(a), f(p))<6 \varepsilon$.

Theorem 7 Let $T$ be an extension of a topology $T_{o}$ on a set $X$. If

(H) $\forall_{A \in T} A \subset \operatorname{Int}_{T_{o}} \mathrm{Cl}_{T_{o}} A$, and

$\left(R_{2}\right) \forall_{U \in T_{o}}$ (the set $\operatorname{Int}_{T_{o}} \mathrm{Cl}_{T_{o}} U$ is T-weak),

then

(C) $C\left(X, T_{o}\right)=C(X, T)$. 
Proof. It is enough to substitute that portion of the proof of Theorem 6 given after (1) with the following:

The set $\operatorname{Int}_{T_{o}}\left(\mathrm{Cl}_{T_{o}} A_{p} \cap \mathrm{Cl}_{T_{o}} A_{a}\right)$ is $T$-open and nonempty, so there is a point

$$
y \in \operatorname{Int}_{T_{o}} \mathrm{Cl}_{T_{o}}\left(\operatorname{Int}_{T_{o}} \mathrm{Cl}_{T_{o}} A_{p} \cap \mathrm{Cl}_{T_{o}} A_{a}\right)
$$

such that no neighborhood of $y$ is $T$-significant. Then, taking $B_{y}=A_{y} \cap$ $\operatorname{Int}_{T_{o}} \mathrm{Cl}_{T_{o}}\left(\operatorname{Int}_{T_{o}} \mathrm{Cl}_{T_{o}} A_{p} \cap \mathrm{Cl}_{T_{o}} A_{a}\right)$, it is enough to notice that it satisfies condition (6) from the proof of Theorem 6.

Theorem 8 Let $T$ be an extension of topology $T_{o}$ in a set $X$. If

(H) $\forall A \in T \quad A \subset \operatorname{Int}_{T_{o}} \mathrm{Cl}_{T_{o}} A$

(R) $\forall A \in T$ the set $\operatorname{Int}_{T_{o}} \mathrm{Cl}_{T_{o}} A$ is T-weak,

then

(C) $C\left(X, T_{o}\right)=C(X, T)$.

Proof. The preceding theorem implies that condition $(R)$ implies condition $\left(R_{2}\right)$.

Definition $4 A$ point $x \in X$ with topology $T$ is said to be a point of local $T$ connectedness if for every $T$-neighborhood $A_{x}$ of $x$, there exists a $T$-connected set $C$ such that $x \in \operatorname{Int}_{T} C \subset C \subset A_{x}$ ).

Corollary 1 Let $T$ be an extension of the natural topology $T_{o}$ in an interval $X \subset \mathbb{R}$. If

(H) $\forall \forall_{A \in T} \quad A \subset \operatorname{Int}_{T_{o}} \mathrm{Cl}_{T_{o}} A$

and

(P) every nonempty $T$-open set $U \subset$ Int $_{T_{o}} \mathrm{Cl}_{T_{o}} U$ contains a point of local T-connectedness.

then

(C) $C\left(X, T_{o}\right)=C(X, T)$.

Proof. First we show that each point of local $T$-connectedness is a $T$-local point. Let $p \in X$ be a point of local $T$-connectedness and let $A_{p}$ be an arbitrary $T$-neighborhood of $p$. It follows from $(P)$ that there exists a $T$-connected set $C$ such that $p \in \operatorname{Int}_{T} C \subset C \subset A_{p}$. As $C$ is $T$-connected, it is $T_{o}$-connected, 
and for the connected subsets of the set of the real numbers with the natural topology we have that $\operatorname{Int}_{T_{o}} \mathrm{Cl}_{T_{o}} C \subset C$. From this and assumption $(H)$ we have

$$
\begin{aligned}
& p \in \operatorname{Int}_{T} C \subset \operatorname{Int}_{T} \mathrm{Cl}_{T} C \subset \operatorname{Int}_{T_{o}} \mathrm{Cl}_{T_{o}} \mathrm{Int}_{T} \mathrm{Cl}_{T} C \subset \\
& \subset \operatorname{Int}_{T_{o}} \mathrm{Cl}_{T_{o}} C \subset C \subset A_{p} \subset \mathrm{Cl}_{T} A_{p} .
\end{aligned}
$$

Hence, $p$ is a $T$-local point, and assumption $(P)$ can be interpreted in the following way.

"Every nonempty $T_{o}$-open set $U \in T_{o} \subset \operatorname{Int}_{T_{o}} \mathrm{Cl}_{T_{o}} U$ contains a T-local point."

But, by Lemma 3 this condition is equivalent to $\left(R_{1}\right)$, and it now follows from Theorem 7 and $(H)$ that $C\left(X, T_{o}\right)=C(X, T)$.

Corollary 2 Let $T$ be an extension of the natural topology $T_{o}$ in an interval $X \subset \mathbb{R}$. If

(H) $\forall_{A \in T} \quad A \subset \operatorname{Int}_{T_{o}} \mathrm{Cl}_{T_{o}} A$,

(S) $T$ does not diminish the class of $T_{o}$-connected sets, and

$\left(P^{\prime}\right)$ for an arbitrary nonempty set $U \in T_{o}$, there exists a point of a local $T_{o}$-connectedness in $\operatorname{Int}_{T_{o}} \mathrm{Cl}_{T_{o}} U$,

then

(C) $C\left(X, T_{o}\right)=C(X, T)$.

Proof. Using assumption $(S)$ and the fact that $\operatorname{Int}_{T_{o}} C \subset \operatorname{Int}_{T} C$ this proof follows the same lines as that of Corollary 1.

The following theorem was proved in [7].

Theorem 9 Let $T$ be an extension of the natural topology $T_{o}$ on an interval $X \subset \mathbb{R}$. If

(W') $\forall_{x \in X} \forall_{A_{x} \in T} \exists_{U_{x} \in T_{o}}\left(U_{x} \backslash A_{x}\right)_{T}^{\prime}=\emptyset$ (where $\left(U_{x} \backslash A_{x}\right)_{T}^{\prime}$ denotes the set of points of $T$-accumulation of the set $\left.U_{x} \backslash A_{x}\right)$,

then

(C) $C\left(X, T_{o}\right)=C(X, T)$. 
Now we will give examples illustrating the lack of interdependence of Theorems $5,6,7,8$ and 9 . In the examples to follow, we adopt the following notation. Let $\mathbb{Q}$ denote a set of rational numbers, and $T_{o}$ the natural topology on $\mathbb{R}$. If $a, b \in \mathbb{R}$, define $a \rho b \Leftrightarrow a-b \in \mathbb{Q}$. It is easy to see that $\rho$ is an equivalence relation and for $a \in \mathbb{R}$ we let [a] denote the equivalence class of $a$.

Example 2 Let $P=\left\{U \backslash \bigcup_{a \in S}[a]: U \in T_{o}, S \subset \mathbb{R}\right.$ is finite or empty $\}$.

It is easy to verify that $P$ is a base of a topology, $T$ on $\mathbb{R}$. It is also easy to see that $T$ is an extension of $T_{o}$ and it can be shown in an elementary way that the topologies $T_{o}$ and $T$ satisfy conditions $(H)$ and $(K)$. By Theorem 5 , $C(\mathbb{R}, T)=C\left(\mathbb{R}, T_{o}\right)$. However, neither condition $\left(P^{\prime}\right)$ nor $\left(W^{\prime}\right)$ is satisfied; so Theorems 8 and 9 do not apply.

Example 3 Let $L=\left\{U \cap[a]: U \in T_{o}, a \in \mathbb{R}\right\}$.

Again, it is easy to verify that the family $T_{o} \cup L$ is a base of a topology $T$ on $\mathbb{R}$ and that $T$ is obviously an extension of the topology $T_{o}$. It is easy to show that conditions $(H),(S)$ and $\left(P^{\prime}\right)$ are satisfied by both $T_{o}$ and $T$. Thus, from Corollary 2, $T$ satisfies $(C)$. However, neither condition $(K)$ nor $\left(W^{\prime}\right)$ is satisfied; so $(C)$ cannot be inferred from Theorems 5 and 9 .

In paper $[6]$ the following theorem is proved.

Theorem 10 Let $\left(X, T_{o}\right)$ be a first countable $T_{31 / 2}$-space. Then

(C) $C\left(X, T_{o}\right)=C(X, T)$ where $T_{o} \subset T$

if and only if

(K) $\forall A \in T \quad A \subset \operatorname{Int}_{T_{o}} \mathrm{Cl}_{T} A$.

Corollary 3 Let $T_{o}$ be the natural topology on $\mathbb{R}$ and let $T \supset T_{o}$. Then

(C) $C\left([a, b], T_{o}\right)=C([a, b], T)$

if and only if

(K) $\forall$ AET $A \subset \operatorname{Int}_{T_{o}} \mathrm{Cl}_{T} A$.

This corollary shows the structure of $T$-open sets satisfying the condition $C\left([a, b], T_{o}\right)=C([a, b], T)$. 


\section{Countably Pseudocompact Spaces}

In 1948 E. Hewitt introduced the following notion of a pseudocompact space.

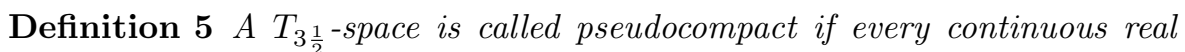
valued function on $\underset{X}{X}$ is bounded.

As has been mentioned before, the subject matter of this paper derives from comparing the classes of the continuous functions with respect to different topologies. The first significant result was the following theorem $([3])$.

Theorem 11 Let $\left(X, T_{o}\right)$ be a compact metrizable space and let $T_{o} \subset T$. Then a necessary and sufficient condition for $C\left(X, T_{o}\right)=C(X, T)$ is that every $T$ continuous function is bounded.

In light of Theorem 11, it was natural to begin an in depth study of the spaces in which all continuous functions are bounded. To this end, the following definition and theorem were given in [3].

Definition 6 A topological space is called a $\star$-compact space if for every pair of families of sets $\left\{F_{y}\right\}$ and $\left\{G_{y}\right\}$ indexed by real numbers $y \geq y_{o}$, satisfying the conditions.

$1^{o} F_{y}$ is closed, $G_{y}$ is open for $y \geq y_{o}$,

$2^{o} y_{o} \leq y_{1} \leq y_{2} \Rightarrow F_{y_{1}} \supset G_{y_{1}} \supset F_{y_{2}} \supset G_{y_{2}}$, and

$3^{o} \quad F_{y} \neq \emptyset \neq G_{y}$ for $y \geq y_{o}$.

The following condition also holds.

$4^{o} \bigcap_{y \geq y_{o}} F_{y}=\bigcap_{y \geq y_{o}} G_{y} \neq \emptyset$.

Theorem 12 A topological space $X$ is $\star$-compact if and only if every real continuous function on $X$ is bounded.

Attempts at characterizing $\star$-compactness by means of notions connected with covering with the open sets or centered families of closed sets have not been successful. Some progress has been made, however, using the notion of a totally (functionally) open set.

Definition 7 A subset $G$ of a topological space $X$ is called totally (or functionally) open if it is of the form $G=f^{-1}(U)$, where $f$ is a continuous real valued function defined on $X$ and $U$ is an open subset of the real line. The complement of a totally open subset of $X$ is called totally closed. 
It is easy to see that if $G$ is totally open, then there exists a continuous function $g: X \rightarrow[0,1]$ such that $G=g^{-1}((0, \infty))$.

In [5], two characterizations of $\star$-compactness were proved which are completely analogous to known characterizations of pseudocompactness. These are below.

Theorem 13 The following conditions are equivalent for every topological space $X$.

1. A space $X$ is pseudocompact (respectively, $\star$-compact) space.

2. Every locally finite family of nonempty open (totally open) subsets of $X$ is finite.

3. Every locally finite, open (totally open) cover of the space $X$ consisting of nonempty sets is finite.

4. Every locally finite, open (totally open) cover of the space $X$ has a finite subcover.

Theorem $\mathbf{1 3}^{\prime}$ The following conditions are equivalent for every topological space $X$.

1. A space $X$ is pseudocompact (respectively, $\star$-compact).

2. For every decreasing sequence $G_{1} \supset G_{2} \supset G_{3} \ldots$ of nonempty open (totally open) subsets of $X, \bigcap_{n=1}^{\infty} \mathrm{Cl} G_{n} \neq \emptyset$.

3. For every countable centered family $\left\{G_{n}\right\}_{n=1}^{\infty}$ of open (totally open) subsets of $X, \bigcap_{n=1}^{\infty} \mathrm{Cl} G_{n} \neq \emptyset$.

In [9] the following definitions were adopted.

Definition 8 A topological space $X$ is called a pseudocompact (countably pseudocompact) space if every (countable) totally open cover of $X$ has a finite subcover.

It is clear that this definition of pseudocompact is equivalent to Hewitt's original (See Definition 5.) in the case that $X$ is $T_{3 \frac{1}{2}}$. In [9], the following was proved.

Theorem 14 A topological space, $X$, is countably pseudocompact if and only if it is $\star$-compact. 
Proof. First, suppose $X$ is countably pseudocompact and let $f$ be an arbitrary continuous real valued function on $X$. Then $X=\cup_{k=1}^{\infty} G_{k}$, where $G_{k}=f^{-1}((k, k+2))$. The cover $\left\{G_{k}\right\}_{k=1}^{\infty}$ is countable and totally open; so there exists a finite set $\left\{k_{1}, k_{2}, \ldots, k_{N}\right\}$ such that $X=\cup_{i=1}^{N} G_{k_{i}}$. Hence, $f(X)=\cup_{i=1}^{N}\left(k_{i}, k_{i}+2\right)$ and so, $f$ is bounded on $X$, and as $f$ is arbitrary, it follows that $X$ is $\star$-compact.

Now assume that $X$ is not countably pseudocompact. Then there exists a totally open covering $\left\{U_{n}\right\}_{n=1}^{\infty}$ of the space $X$ which contains no finite subcover. If $G_{n}=\cup_{i=1}^{n} U_{i}$, then $\left\{G_{n}\right\}_{n=1}^{\infty}$ is an increasing sequence of totally open sets whose union is $X$. Indeed, we may assume this sequence to be strictly increasing so that for each $n, G_{n+1} \backslash G_{n} \neq \emptyset$.

The sets $G_{n}$ are totally open. Thus

$$
\forall_{n \in N} \exists_{g_{n}} \text {-continuous on } X \exists_{a_{n}} G_{n}=\left\{x \in X: g_{n}(x)<a_{n}\right\} \text {. }
$$

Let $h_{n}(x)=\max \left[0, a_{n}-g_{n}(x)\right]$. Then

(i) for $x \in G_{n}$ we have $h_{n}(x)>0$

(ii) for $x \in X \backslash G_{n}$ we have $h_{n}(x)=0$.

Now set $f_{n}(x)=\min \left\{2^{-n}, h(x)\right\}$ and let $\phi(x)=\sum_{n=1}^{\infty} f_{n}(x)$. Then $\phi$ is continuous and positive at each $x \in X$ and hence, $f(x)=\frac{1}{\phi(x)}$ is also positive and continuous at each $x \in X$

Since the $G_{n}$ are distinct, it follows that

$$
\forall_{n \in N} \exists_{x_{n} \in G_{n+1} \backslash G_{n}} f_{1}\left(x_{n}\right)=\ldots=f_{n}\left(x_{n}\right)=0 .
$$

But $0<f_{n+k}\left(x_{n}\right) \leq 2^{-n-k}$ for $k=1,2, \ldots$, so that

$$
\phi\left(x_{n}\right)=\sum_{n=1}^{\infty} f_{n+k}\left(x_{n}\right) \leq \sum_{n=1}^{\infty} 1 / 2^{n+k}=1 / 2^{n} .
$$

Hence, for each $n, f\left(x_{n}\right) \geq 2^{n}$. The fact that $f$ is continuous but not bounded completes the proof that $X$ is not $\star$-compact.

Definition 8 is clearly the direct analogue of the definition of compact (countably compact). In order to emphasize the analogy further, we will omit axiom $T_{2}$ from the usual definitions of the latter.

Definition 9 A topological space $X$ is called a compact (countably compact) space if every (countable) open cover of $X$ has a finite subcover. 
These analogies are not confined to the definitions but carry over to the basic properties as well. This is shown in the following two theorems.

\begin{tabular}{|c|c|}
\hline Theorem 15 & Theorem $15^{\prime}$ \\
\hline 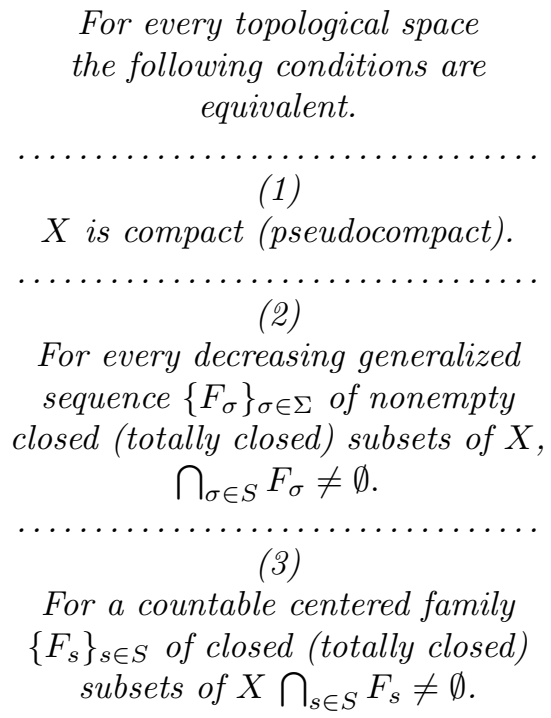 & 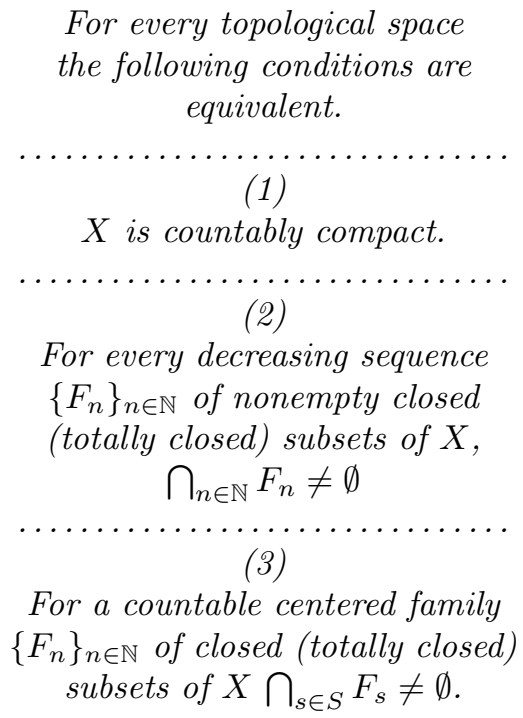 \\
\hline
\end{tabular}

The dependencies among these four classes of topological spaces are presented in the following diagram.

Compact spaces $\downarrow$

Pseudocompact spaces $\longrightarrow$

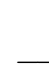

\section{Countably compact spaces}

\section{$\downarrow$}

\section{Countably pseudocompact spaces}

\section{On Locally Uniformly Converging Sequences}

In [4], three theorems are given which contain characterizations of compactness, countable compactness and countable pseudocompactness. These characterizations depend on the connections between the notions of locally uniform convergence and uniform convergence defined below.

Definition 10 A sequence $\left\{f_{n}\right\}$ of real functions which converges to a function $f$ is said to be locally (totally) uniformly convergent in a topological space $X$, if for every point $x_{o} \in X$ there exists a neighborhood $U\left(x_{o}\right)$ (totally open) of $x_{o}$ such that $\left\{f_{n}\right\}$ converges to $f$ uniformly in $U\left(x_{o}\right)$. 
In [7], an analogous theorem is given which contains characterizations of pseudocompactness. All four theorems are given below.

\begin{tabular}{|c|c|}
\hline Theorem 16 & Theorem 16' \\
\hline $\begin{array}{c}\text { A topological space } X \text { is } \\
\text { compact if and only if every } \\
\text { locally uniformly convergent, } \\
\text { generalized sequence of functions } \\
\text { is uniformly convergent. }\end{array}$ & $\begin{array}{c}\text { A topological space } X \text { is } \\
\text { countably compact if and only } \\
\text { if every locally uniformly convergent } \\
\text { sequence of functions is uniformly } \\
\text { convergent. }\end{array}$ \\
\hline Theorem 17 & Theorem 17' \\
\hline $\begin{array}{l}\text { A topological space } X \text { is pseudo- } \\
\text { compact if and only if every locally } \\
\text { totally uniformly convergent, } \\
\text { generalized sequence of functions } \\
\text { is uniformly convergent. }\end{array}$ & $\begin{array}{c}\text { A topological space } X \text { is countably } \\
\text { pseudocompact if and only if every } \\
\text { locally totally uniformly convergent } \\
\text { sequence of functions is uniformly } \\
\text { convergent. }\end{array}$ \\
\hline
\end{tabular}

Proof of Theorem 16. Suppose that $X$ is not compact and let $\mathcal{G}$ be a family of open subsets of $X$ such that no finite subfamily covers $X$. Let $\Sigma$ be the set of finite sums $\bigcup_{i=1}^{k} G_{i}$, where $G_{i} \in \mathcal{G}$. For $\sigma=\bigcup_{i=1}^{k} G_{i}$ and $\sigma^{\prime}=\bigcup_{i=1}^{l} G_{i}^{\prime}$ belonging to $\Sigma$ put $\sigma \leq \sigma^{\prime}$ if and only if $\bigcup_{i=1}^{k} G_{i} \subset \bigcup_{i=1}^{l} G_{i}^{\prime}$. The set $\Sigma$ is thus directed by the relation " $\leq$ ". We have $X=\bigcup_{\sigma \in \Sigma} \sigma$, but $X \neq \sigma$ for every $\sigma \in \Sigma$. Put

$$
f_{\sigma}(x)=\left\{\begin{array}{lll}
1 & \text { for } & x \in \sigma \\
0 & \text { for } & x \notin \sigma
\end{array} .\right.
$$

The sequence $\left\{f_{\sigma}\right\}_{\sigma \in \Sigma}$ converges to $f(x) \equiv 1$ locally uniformly, but this convergence is not uniform.

Conversely, suppose that $X$ is pseudo-compact, and let $\left\{f_{\sigma}\right\}_{\sigma \in \Sigma}$ be an arbitrary generalized sequence converging locally uniformly to some function $f$. We shall prove that $\left\{f_{\sigma}\right\}_{\sigma \in \Sigma}$ converges to $f$ uniformly. Let $\varepsilon$ be an arbitrary positive number. For every point $x_{o} \in X$ there exists a neighborhood $U\left(x_{o}\right)$ of $x_{o}$ and $\sigma_{x_{o}} \in \Sigma$ such that for every $x \in U\left(x_{o}\right)$ and every $\sigma \geq \sigma_{x_{o}}$ we have $\left|f_{\sigma}(x)-f(x)\right|<\varepsilon$. The family, $U$, of such neighborhoods $U(x)$ for $x \in X$ is a cover of $X$, and by compactness, $U$ contains a finite subcover $\left\{U\left(x_{1}\right), \ldots, U\left(x_{k}\right)\right\}$. Since $\Sigma$ is directed, there exists $\sigma^{\prime} \in \Sigma$ such that $\sigma^{\prime} \geq$ $\sigma_{x_{1}}, \ldots, \sigma_{x_{k}}$. Hence,

$$
\forall_{x \in X} \forall_{\sigma \geq \sigma^{\prime}}\left|f_{\sigma}(x)-f(x)\right|<\varepsilon
$$


and $\left\{f_{\sigma}\right\}_{\sigma \in \Sigma}$ converges to $f$ uniformly.

The proof of Theorem $16^{\prime}$ is analogous.

Proof of Theorem 17. Suppose that $X$ is not pseudocompact; i.e. that there exists a family $\left\{G_{s}\right\}_{s \in \mathbb{N}}$ of totally open subsets of $X$ such that for every finite system $\left\{s_{1}, \ldots, s_{k}\right\} \subset \mathbb{N}, X \neq \bigcup_{i=1}^{k} G_{s_{i}}$. Using this, we give an example of a sequence which converges locally uniformly but this convergence is not uniform.

As the sets $G_{s}$ are totally open, for every $s \in \mathbb{N}$ there exists a continuous real valued function $g_{s}$ and a point $a_{s} \in \mathbb{R}$ such that $G_{s}=\left\{x \in X: g_{s}(x)<\right.$ $\left.a_{s}\right\}$. Let $\Sigma$ denote the set of all finite subsets of $\mathbb{N}$, directed by " $\subset$ ". For each $\sigma \in \Sigma$. let $H_{\sigma}=\cup_{j=1}^{n} G_{s_{j}}$, and $h_{\sigma}(x)=\max \left\{0, a_{s_{j}}-g_{s_{j}}(x)\right\}$. Then, $\left\{H_{\sigma}\right\}_{\sigma \in \Sigma}$ is an increasing generalized sequence of totally open sets.

Now consider $T=\Sigma \times \mathbb{N}$. If $\tau^{\prime}=\left(\sigma^{\prime}, k^{\prime}\right), \tau^{\prime \prime}=\left(\sigma^{\prime \prime}, k^{\prime \prime}\right) \in T$, define $\tau^{\prime} \leq$ $\tau^{\prime \prime} \Leftrightarrow \sigma^{\prime} \leq \sigma^{\prime \prime}$ and $k^{\prime} \leq k^{\prime \prime}$. For $\tau \in T$, let $f_{\tau}(x)=\min \left\{1, k \cdot h_{\sigma}(x)\right\}=$ $\min \left\{1, \max _{1 \leq j \leq n}\left\{0, a_{s_{j}}-g_{s_{j}}(x)\right) \cdot k\right\}$, and let let $x_{o} \in X$. There exists a set $G_{s_{o}}$ such that $x_{o} \in G_{s_{o}}$ and $a_{s_{o}}-g_{s_{o}}(x)>0$ Thus, there exists $k_{o} \in N$ such that $\left(a_{s_{o}}-g_{s_{o}}(x)\right) \cdot k>1$. Because the function $\left(a_{s_{o}}-g_{s_{o}}(x)\right) \cdot k$ is continuous at $x_{o} \in G_{s_{o}}$, there exists a totally open neighborhood $U\left(x_{o}\right) \subset G_{s_{o}}$ such that $\left(a_{s_{o}}-g_{s_{o}}(x)\right) \cdot k>1$ for all $x \in U\left(x_{o}\right)$. Then for $\tau_{o}=\left(\sigma_{o}, k_{o}\right)$ and $\sigma_{o}=$ $\left\{s_{o}\right\}$ we have $f_{\tau_{o}}(x)=1$ for $x \in U\left(x_{o}\right)$. Because the sequence $\left\{f_{\tau}\right\}_{\tau \in T}$ is nondecreasing, we have $f_{\tau}(x)=1$ whenever $\tau \geq \tau_{o}$ and $x \in U\left(x_{o}\right)$. Hence, the sequence $\left\{f_{\tau}\right\}_{\tau \in T}$ converges to $f(x)=1$ locally totally uniformly. On the other hand, let $\sigma \in \Sigma$ and $x_{\sigma} \in X \backslash H_{\sigma}$. Because $H_{\sigma}=\bigcup_{j=1}^{n} G_{s_{j}}$, it follows that $\max \left\{0,\left(a_{s_{j}}-g_{s_{j}}(x)\right) \cdot k\right\}=0$, i.e. $f_{\tau}\left(x_{\sigma}\right)=0$.

Hence, for $\varepsilon=1 / 2$ we have

$$
\forall_{\left(\sigma_{o}, k_{o}\right)} \exists_{(\sigma, k)>\left(\sigma_{o}, k_{o}\right)} \exists_{\left\{x_{\sigma}\right\}_{\sigma \in \Sigma}}\left|f_{(\sigma, k)}\left(x_{\sigma}\right)-f\left(x_{\sigma}\right)\right|=1>\varepsilon
$$

and this means that the convergence of the sequence $\left\{f_{\tau}\right\}_{\tau \in T}$ is not uniform.

Conversely, suppose that $X$ is pseudocompact and let $\left\{f_{\sigma}\right\}_{\sigma \in \Sigma}$ be an arbitrary generalized sequence converging locally totally uniformly to some function $f$. We shall prove that $\left\{f_{\sigma}\right\}_{\sigma \in \Sigma}$ converges to $f$ uniformly. Let $\varepsilon$ be an arbitrary positive number. For every point $x_{o} \in X$ there exists a neighborhood $U\left(x_{o}\right)$ of $x_{o}$ and $\sigma_{x_{o}} \in \Sigma$ such that for every $x \in U\left(x_{o}\right)$ and $\sigma \geq \sigma_{x_{o}}$ we have $\left|f\left(x_{\sigma}\right)-f(x)\right|<\varepsilon$. Any family $\{U(x)\}_{x \in X}$ of such neighborhoods is a totally open cover of $X$. Hence, there is a finite subcover $\left\{U\left(x_{1}\right), \ldots, U\left(x_{k}\right)\right\}$ of $X$, and since $\Sigma$ is directed, there exists $\sigma^{\prime} \in \Sigma$ such that $\sigma^{\prime} \geq \sigma_{x_{1}}, \ldots, \sigma_{x_{k}}$. That is,

$$
\forall_{x \in X} \forall_{\sigma \geq \sigma^{\prime}}\left|f_{\sigma}(x)-f(x)\right|<\varepsilon,
$$

and hence, $\left\{f_{\sigma}\right\}_{\sigma \in \Sigma}$ converges to $f$ uniformly. 
Remark 8 In this proof we have not used the continuity of functions $f_{\sigma}$.

Proof of Theorem $17^{\prime}$ (without using the notion of a totally open set). Suppose that $X$ is not countably pseudocompact, and that $\phi: X \rightarrow \mathbb{R}$ is continuous but unbounded. Put $f_{n}(x)=\min \{|\phi(x)|, n\}$ for $n=1,2, \ldots$. Then, $f_{n}$ is continuous on $X$ for each $n=1,2, \ldots$ For each $x_{o} \in X$, there is an $N=N(x) \in \mathbb{N}$ such that $\left|\phi\left(x_{o}\right)\right|<N$. Since $\phi$ is continuous, there is a neighborhood $U\left(x_{o}\right)$ of $x_{o}$ such that for every $x \in U\left(x_{o}\right)$ we have $|\phi(x)|<N$. Hence,

$$
\exists_{U\left(x_{o}\right)} \exists_{N} \forall_{x \in U\left(x_{o}\right)} \forall_{n \geq N} f_{n}(x)=|\phi(x)| .
$$

That is, $\left\{f_{n}\right\}$ converges to $|\phi(x)|$ in $X$ locally uniformly but this convergence is not uniform.

Conversely, suppose that there exists a sequence $\left\{f_{n}\right\}$ of continuous functions which converges locally uniformly to some function $f$, but for which the convergence is not uniform. There exists $\varepsilon_{o}>0$ and a sequence $\left\{x_{n}\right\}$ such that $\left|f_{n}\left(x_{n}\right)-f\left(x_{n}\right)\right|>\varepsilon_{o}$ for $n=1,2, \ldots$. Now, for each $n$, put $\phi_{n}(x)=\min \left\{0, \varepsilon_{o}-\left|f_{n}(x)-f(x)\right|\right\}$. Then, $\phi_{n}$ is continuous on $X$ for all $n \in \mathbb{N}$. Let $x_{o} \in X$. Since $\left\{f_{n}\right\}$ converges to $f$ locally uniformly, there exists a neighborhood $U\left(x_{o}\right)$ of $x_{o}$ and $N>0$ such that for every $x \in U\left(x_{o}\right)$ and $n \geq N$ we have $\left|f_{n}(x)-f_{n}(x)\right|<\varepsilon_{o}$. That is, $\phi_{n}(x) \equiv 0$ for all $x \in U\left(x_{o}\right)$ and all $n>N$. Thus, the function

$$
\phi(x)=\sum_{k=1}^{\infty} \frac{\left|\phi_{k}(x)\right|}{\left|\phi_{k}\left(x_{k}\right)\right|} \cdot k
$$

is continuous on $X$. However, $\phi\left(x_{n}\right) \geq n$ and thus, $\phi$ is unbounded on $X$. Hence $X$ is not countably pseudocompact.

Remark 9 In this proof we have not used local total uniform convergence but only local uniform convergence.

\section{Generalizations of Dini's Theorem}

In this section we investigate the following classical theorem of Dini.

Theorem 15 ([1]) If $X$ is a compact space, then every monotone sequence $\left\{f_{n}\right\}$ of continuous functions which converges to a continuous function $f$, converges uniformly to $f$.

Now we will discuss four generalizations of this theorem characterizing four classes of topological spaces presented in Part 2. 


\begin{tabular}{|c|c|}
\hline Theorem 19 [9] & Theorem $\mathbf{1 9}^{\prime}[\mathbf{1 0}]$ \\
\hline $\begin{array}{c}\text { A topological space } X \text { is compact } \\
\text { if and only if every nondecreasing } \\
\text { generalized sequence of lower }\end{array}$ & $\begin{array}{c}\text { A topological space } X \text { is countably } \\
\text { compact if and only if every } \\
\text { nondecreasing sequence of lower } \\
\text { converges to a continuous function, } \\
\text { is uniformly convergent. }\end{array}$ \\
$\begin{array}{c}\text { semicontinuous functions which } \\
\text { conves to a continuous function, } \\
\text { is uniformly convergent. }\end{array}$ \\
\hline
\end{tabular}

\begin{tabular}{|c|c|}
\hline Theorem 20 [9] & Theorem 20' [10] \\
\hline A topological space $X$ is & A topological space $X$ is \\
pseudocompact if and only & countably pseudocompact if \\
if every monotone generalized & and only if every monotone \\
sequence of continuous functions & sequence of continuous functions \\
which converges to a continuous & which converges to a continuous \\
function, is uniformly convergent. & function, is uniformly convergent. \\
\hline
\end{tabular}

Proof of Theorem 19. First assume that $X$ is a compact space and $\left\{f_{\sigma}\right\}_{\sigma \in \Sigma}$ is an arbitrary generalized nondecreasing sequence of the lower semicontinuous functions, which converges to a continuous function, $f$. Let $\varepsilon>0$ and put

$$
G_{\sigma}=\left\{x \in X: f_{\sigma}(x) \geq f(x)-\varepsilon\right\} .
$$

As $\left\{f_{\sigma}\right\}_{\sigma \in \Sigma}$ is nondecreasing and convergent to $f$, the corresponding sequence of sets $\left\{G_{\sigma}\right\}_{\sigma \in \Sigma}$ is increasing and $X=\bigcup_{\sigma \in \Sigma} G_{\sigma}$. Because the functions $f_{\sigma}-f$ are lower semicontinuous, the sets $G_{\sigma}$ are open. It now follows from the compactness of $X$ that there exists $\sigma_{o} \in \Sigma$ such that $X=G_{\sigma_{o}}$ and hence that

$$
f_{\sigma_{o}}(x)-f(x)>-\varepsilon \quad \text { for every } x \in X .
$$

Thus, by the monotonicity of a sequence $\left\{f_{\sigma}\right\}_{\sigma \in \Sigma}$, we have that $\mid f_{\sigma}(x)-$ $f(x) \mid<\varepsilon$ for every $x \in X$ and $\sigma \geq \sigma_{o}$. That is, the sequence $\left\{f_{\sigma}\right\}_{\sigma \in \Sigma}$ converges uniformly to $f$ and the proof is complete.

We now turn to the general case. There exists a generalized increasing sequence $\left\{G_{\sigma}\right\}_{\sigma \in \Sigma}$, of open sets such that $\cup_{\sigma \in \Sigma} G_{\sigma}=X$ but for each $\sigma \in \Sigma$ and $X \neq G_{\sigma}$. Let $\sigma \in \Sigma$ and define

$$
f_{\sigma}(x)=\left\{\begin{array}{ll}
1 & \text { for } x \in G_{\sigma} \\
0 & \text { for } x \notin G_{\sigma}
\end{array} .\right.
$$


Then $f_{\sigma}$ is lower semicontinuous and $\left\{f_{\sigma}\right\}_{\sigma \in \Sigma}$ is a nondecreasing sequence converging to $f(x) \equiv 1$. However, this convergence is not uniform.

The proof of Theorem $19^{\prime}$ is analogous.

Proof of Theorem 20. First assume that $X$ is pseudocompact, $\left\{f_{\sigma}\right\}_{\sigma \in \Sigma}$, is an arbitrary generalized, monotone sequence of continuous functions, converging to a continuous function $f$. Let $\varepsilon>0$ and put

$$
G_{\sigma}=\left\{x \in X:\left|f_{\sigma}(x)-f(x)\right|<\varepsilon\right\}
$$

Since the sequence is monotone and converges to $f$, the sequence of the sets $\left\{G_{\sigma}\right\}_{\sigma \in \Sigma}$, is increasing and $X=\bigcup_{\sigma \in \Sigma} G_{\sigma}$. Because the functions $\left|f_{\sigma}-f\right|$ are continuous, the sets $G_{\sigma}$ are functionally open, and since $X$ is pseudocompact, it follows that there exists $\sigma_{o} \in \Sigma$ such that $X=G_{\sigma}$. Hence $\mid f_{\sigma_{o}}(x)-$ $f(x) \mid<\varepsilon$ for $x \in X$, and thus by the monotonicity of the sequence $\left\{f_{\sigma}\right\}_{\sigma \in \Sigma}$, we have $\left|f_{\sigma_{o}}(x)-f(x)\right|<\varepsilon$ for $x \in X$ and $\sigma \geq \sigma_{o}$. Hence, $\left\{f_{\sigma}\right\}_{\sigma \in \Sigma}$, is uniformly convergent to $f$. Now suppose $X$ is not pseudocompact. Then there exists a family of functionally open sets $\left\{G_{s}\right\}_{s \in \mathbb{N}}$, such that $X=\bigcup_{s \in \mathbb{N}} G_{s}$, but $X \neq \bigcup_{j=1}^{k} G_{s_{j}}$ for every finite system $s_{1}, s_{2}, \ldots, s_{k} \in \mathbb{N}$. The sequence $\left\{f_{\tau}\right\}_{\tau \in T}$ described in the proof of Theorem 17 is nondecreasing and converges to $f(x) \equiv 1$, but this convergence is not uniform.

Proof of Theorem 20' (without using the notion of a totally open set). Assume that $X$ is not countably pseudocompact. Then there is a continuous real valued function, $\phi$, defined on $X$ which is not bounded. Thus, if

$$
f_{n}(x)=\min \{n,|\phi(x)|\}
$$

then $\left\{f_{n}(x)\right\}$ is a monotone sequence of continuous real valued functions on $X$, which converges to

$$
f(x)=|\phi(x)| \text { for every } x \in X .
$$

However, this convergence is not uniform.

Now suppose $X$ is countably pseudocompact and $\left\{f_{n}\right\}$ is a monotone sequence of continuous real valued on $X$, which converges to a continuous function $f$. We will show that the sequence $\left\{f_{n}\right\}$ is uniformly convergent. Without loss of generality, we can assume that $\left\{f_{n}\right\}$ is decreasing to the zero function. This is because $\left\{\left|f_{n}-f\right|+1 / n\right\}$ decreases to zero and is uniformly convergent if and only if $\left\{f_{n}\right\}$ converges to $f$ uniformly. Let $\varepsilon>0$ for and $n \leq y \leq n+1$, put

$$
F_{y}=\left\{x \in X:(y-n) f_{n+1}(x)+(1-(y-n)) f_{n}(x) \geq \varepsilon\right\}
$$


and

$$
G_{y}=\left\{x \in X:(y-n) f_{n+1}(x)+(1-(y-n)) f_{n}(x)>\varepsilon\right\}
$$

For $y_{o}=1$, the sets $F_{y}, G_{y}$ satisfy, conditions $1^{\circ}$ and $2^{\circ}$ from Definition 6 , but do not satisfy condition $4^{\circ}$. So condition $3^{\circ}$ from that Definition 6 cannot be satisfied. Thus there exists a number $y *$ such that $F_{y *}=\emptyset$. So we have $F_{y}=\emptyset$ for $y \geq y *$. If $n_{o}$ is the least integer exceeding $y *$, we have that for $n \geq n_{o}$,

$$
F_{n}=\left\{x \in X: f_{n}(x) \geq \varepsilon\right\}=\emptyset .
$$

that is, for every $x \in X,\left|f_{n}(x)\right|<\varepsilon$ for $n \geq n_{o}$ and hence, the sequence $\left\{f_{n}\right\}$ converges uniformly.

\section{References}

[1] R. Engelking, General topology, Polish Scientific Publishers, Warszawa 1977.

[2] W. Grudziński, Topological extensions not changing the class of continuous functions, Doctoral Thesis, Institute of Mathematics, Technical University of Łódź, Łódź 1983.

[3] E. Kocela, Properties of some generalizations of the notion of continuity of a function, Fund. Math. LXXVIII (1973), 133-139.

[4] B. Koszela, On locally uniformly converging sequences, Scientific Bulletin of Łódź Technical University, Matematyka, 11 (1978), 11-15.

[5] B. Koszela, On some characterization of $\star$-compact spaces, Scientific Bulletin of Łódź Technical University, Matematyka, 11 (1978), 17-21.

[6] B. Koszela, On some condition of the equality of classes of continuous functions with different topologies, Dem. Math., X (1977), 617-627.

[7] B. Koszela, On some characterization of chosen types of compactness, Scientific Bulletin of Lodz Technical University, Matematyka, 27 (1995), $75-79$.

[8] B. Koszela, T. Świątkowski, and W. Wilczyński, Classes of continuous real functions, Real Anal. Exch., 4, (1978-79), 139-157.

[9] E. Smach-Jóźwik, Theorem of Dini for nets, Doctoral Thesis, Institute of Mathematics, Technical University of Łódź, Łódź, 1981.

[10] T. Świagtkowski, On some theorem of Dini' Scientific Bulletin of Lodz University, Matematyka, 52 (1973), 53-56. 